\title{
RIOBAMBAPI KAPAK KAPAKKUNAPAK WAWA YALLIRI RAYMIPI RIKCHAY SUPAY ANTA
}

\author{
LA FIGURA DEL DIABLO DE LATA EN EL PASE DEL NIÑO REY DE REYES DE \\ RIOBAMBA
}

\section{THE FIGURE OF THE DEVIL OF LATA IN THE PASS OF THE CHILD KING OF KINGS OF RIOBAMBA}

\section{UCHILLA YUYAYKUNA}

Kay llankaypika Supay Anta Wawa Kapak Kapakkunapak Riobamba puri raymikuna tiyashkatami runakunapak kawsaywan taripashka hamutarikrinchik. Shuknikipika, chimpapurashpami rikushun maypi Supaypak yuyay kallarishkata, ashtawanka España raymikunapi rikushkata chimpapurashun, crishkawan yuyarishpa, Abya Yalapika rikushun colonizadores shinallatak cristianismowan chayamushkata. Kipapika parlanakushkakunawan hamutarishun, imashina Riobamba markapika kallarirka kay raymikunawan, shinallatak imashina ñawpakpan mana kunkarishpa wiñayta paktashpa katichun. Kipa tantachishka yuyaypika chayashun ima shina mishukunaka ñawpakman apakunkuna raymikunata, kay paktaykunaka rikuchinmi ima shina Wawa puri raymikunapi wakichikta, ashtawankarin runakunapak yuyayta mana chinkachispa katinapak.

Rikuy Yuyaykuna: Antapak supay, kapak wapak yalliri, Riobamba, shuktikrikuna.

\section{RESUMEN}

El presente trabajo de investigación analiza las características culturales y sociales del Diablo de Lata en el Pase del Niño Rey de Reyes de Riobamba (Ecuador). En primer lugar, se hace un recorrido comparativo con los orígenes de la figura del diablo en las celebraciones en España de esta figura y su difusión, mediante sincretismo religioso, por América con la llegada de los colonizadores y el cristianismo. A continuación, mediante la etnografía enfocada no participativa y la entrevista, se indaga en el origen de la figura en Riobamba y sus características formales, para definirlas y preservarlas. Se llega a la conclusión de que es una figura fruto del mestizaje y del sincretismo, que su función es proteger al Niño en el pase y que por tanto responde a un equilibrio de fuerzas propio de la cosmovisión andina, con sus características primigenias y actuales, fruto de la evolución del personaje.

Palabras clave: Diablo de Lata, Pase del Niño, Riobamba, aculturación.

\section{ABSTRACT}

This research paper analyzes the cultural and social characteristics of the Devil of Lata in the parade of the Child King of Kings of Riobamba (Ecuador). In the first place, a comparative tour is made with the origins of the figure of the devil in the celebrations in Spain of this figure and its diffusion, through religious syncretism, through America with the arrival of the colonizers and Christianity. Then, through the non-participatory focused ethnography and the interview, the origin of the figure in Riobamba and its formal characteristics are investigated, to define and preserve them. It is concluded that it is a fruit of miscegenation and syncretism, that its function is to protect the Child in the parade and therefore responds to a balance of typical forces of the Andean worldview, with its original and current characteristics, the fruit of the evolution of the character.

Keywords: Devil of Tin, Parade of the Child, Riobamba, acculturation.

\section{Jennifer M. Loaiza Peñafiel}

marjenn0320@gmail.com

Universidad Nacional de Chimborazo, Grupo de Investigación Puruhá, Riobamba, Ecuador,

ORCID: https://orcid.org/0000-0001-7065-1456

\section{Miryam Cecilia Mora Cayambe}

miryamleo18@live.com

Universidad Nacional de Chimborazo, Grupo de Investigación Puruhá, Riobamba, Ecuador,

ORCID: https://orcid.org/0000-0003-1054-9294 


\section{KALLARIYUYAY}

Kay llankaypika taripashkami ashka minishtirishkata rikushpa yuyarinkapak Supaypak churarita, Supay tukushka runakunaka Wawa Kapak Kapakkunapak puri raymipika mana illak kana kashkata. Watan watan kunatami kay rikuchikunaka shamushka, shinapish ashtawan allichirishka kan punchanta shukta rikuy kunawan, kay tukuy yuyaykunaka shamushlami llaktapi kawsay runakunamanta, kunanka tukushkami may sumak rikuchi Colonia punchakunamanta iglesia Católica ukupipish.

Supay Anta tukushkakunaka Wawa Kapak Kapakkunapak puri raymi punchakunapi tiyankapakka, shamushkami ayllu llaktapi kawsay runakunamanta (Diablo Indígena) kayshuk rikuchikunaka watanta raymikunamantami allichirishpa shamushka. Maypika ashka sapi yuyaykunapish chinkarishkami, churaymanta rikushpa shinallatak tushuy kunamantapish.

Kay Waka Kapak Kapakkunapak puri raymi paktanmi Riobamba quiti uku ñankunata, kallarinmi Santa Rosamanta purishpa Iglesia de San Antonio (Loma de Quitokama) chaypimi sumak misata paktachinkuna, chay kipaka tukuy tushukkunawan kutinkuna maymanta kallarishkaman chaypi tukuchinkapak raymikunata. Tukuymanta yalli sumak rikunaka kanmi Wawa Kapak Kapakkunapak shuyuta, paywan rikkunatapish, takikkuna, asichikkuna, kushullukuna, supay anta tukushkukuna, kurikinkikuna, sacha runakuna.

Supay anta tukushka tushukkunami ñawpashpa rinkuna Wawa Kapak Kapakkunapak shuyu katichunka, shinallatak charinmi samaypak paktaytapish. Imashina shuktak shuktak llaktakunapi kallarishkata rikukpika, rikunchikmi ñawpa pachakunamanta wiñarishkatami yachakchayanchik (origen prehispánico) runapak kawsaypika kay raymikunaka paktanmi tukuy kay pachami imalla tiyashkakunawan tanllata sumakta kawsana kashkata rikuchinapak.

Maypika sinchimi kashka maymanta wiñarishkata riksik chayanaka, ima yuyaykunawan wiñarishkatapish, shinapish Riobambeño kunapakka may sumay rikuymi kan Wawa Kapak Kapakkunapak purikunapika, shuktak karu llaktapi tiyak raymikunawan rikukpika watantami allichirishpa shamushka rikurin.

Tukuy kay Supay rikuyka rikurinmi España raymikunamanta, punta rikuyka rikurishkami waranka - patsak - pichka chunka (1150) watakunapi Barcelona llaktapi, chaypimi Ramón Berenguer IV paypak Aragón ñusta Petronilawan raymita charishkapi. Waranka - pichka patsak - iskun chunka - pichka (1595) watakunapika rikurishkami Melchor Machado El Dragón del Curpus raymikunata rurashkapi, chaipimi chay hatun supay tukushkaka rikuchishka. Chay kipaka tukuy Europa llaktakunapi kay ruraykunawanka katishkakuna (Ramírez 2018).

"Tajo mayu kuchukunapika siglo XIV, manarik Cristobal Colón América allpakunaman chayamukpimi kay raymikunataka rurakkuna kashka Corpus Christi punchakunapi, chaymantami iglesiapika tukuy chay rurakkunata wiñachishpa churashka, Europa shinallatak mushuk Abya Yala llaktakunapipish" (Irady 2018).

Karuespañamanta shuwa runakunaAbyaYala llaktaman chayamushka kipa shinallatak alli willay runakunaman chayamushkamantami, kay raymikunaka ashtawan allichirishpa kallarishka, runakunapak raymi ruraykunawan tantachishpa. Shinapish ashka llakikunatami apamushka kay tantachi ruraykunawanka kay mamallaktakunapi: México, Cuba, Bolivia, Perú, Venezuela, Colombia shinallatak nukanchik Ecuador mama llaktapipish.

Abya Yaya chawpi shinallatak kullasuyu llaktakunapika ñami charirkakuna sumak kawsayta, raymikunata, tushuykunata manarak Españolkuna chayamukpi. Españolkuna chayamushkamantami 


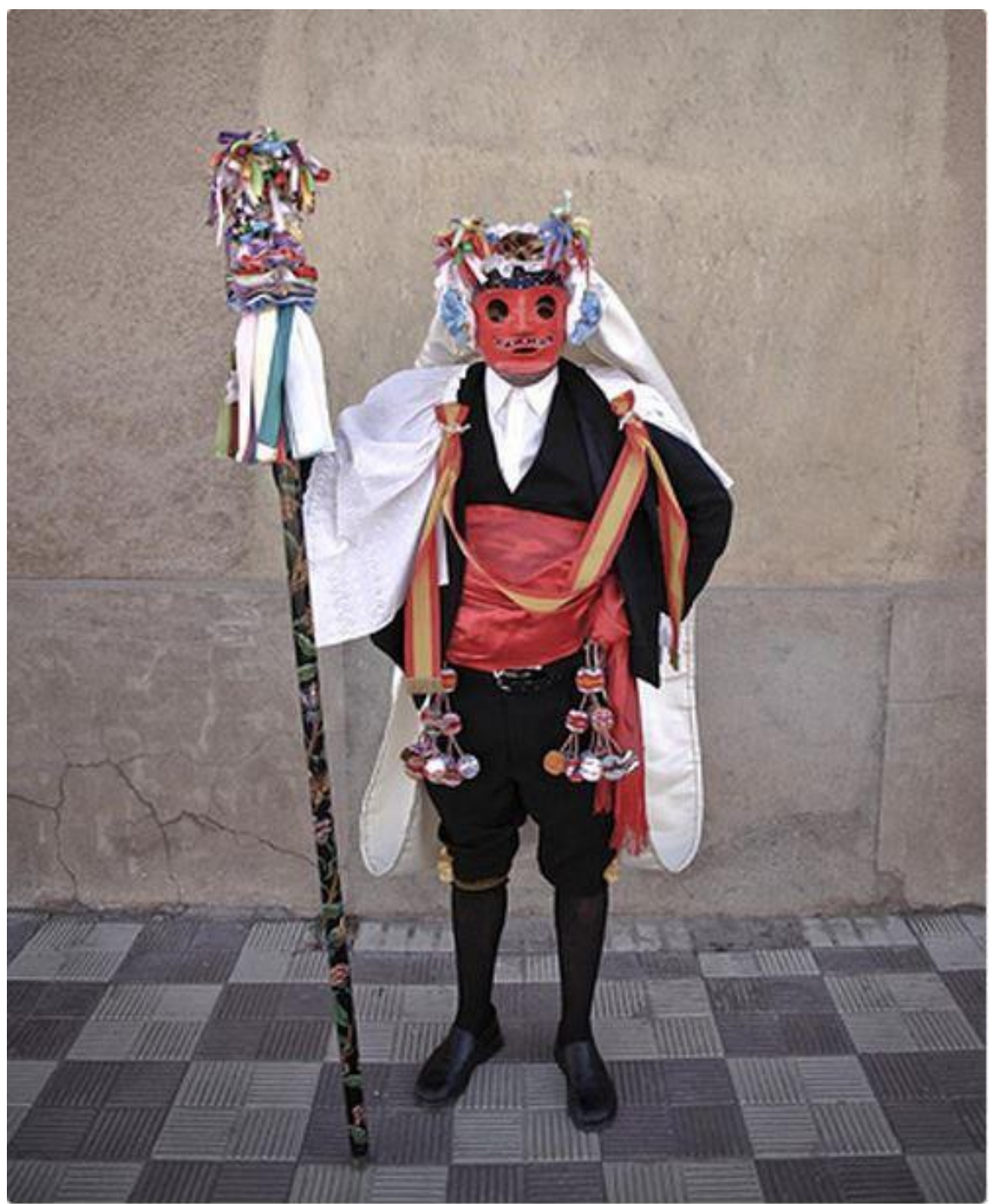

Shuyu 1. Pecados de Camuña (Toledo, España).

chakrurishpa shamushka llakikunata apashpa.

Ecuador mama llaktapika tawka raymikunami tiyan, ñawpa yuyaknunamanta pachami sapiyashpa shamushpa shuktak shuktak ruraykunawan, kay raymikunaka mirarishpami katishka Españolkuna chayamukpipish, ashtawanpish shukllami tukushka runakunapak yuyaywan mishukunapak yuyaywan.

Wawa puri raymika Riobamba kitipika kanmi hatun raymikunapak ukupi, chushku pas- tak (400) wawa pury raymukunami yallirin Chimborazo marka ukupika samay - kulla killakunapi. Chaymantami ishki waranka chunka pusak (2018) watapi, kuski killa chunka punllakunapi kay hatun raymika $\mathrm{Pa}$ trimonio Cultural Inmaterial del Ecuador ukupi yaykushka.

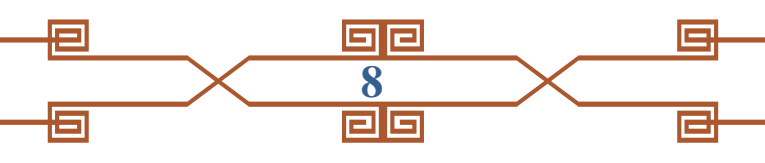




\section{ECUADOR MAMALLAKTAPI, SU- PAYKUNA SHUKTAK RAYMIKUNAPI}

"Supay tukushkakunaka manatak illaytukunchu ashka raymikunapi tukuy Ecuador mamallakta ukupitak. Ayllu llaktakunapipish, shuktak shina churaykunawan shinallatak shuktak tushuykunawan paktashpa rikurinmi." (Diablos: símbolo de la alegría popular 2018).

\section{TUNGURAHUA MARKA, PÍLLARO SUPAYKUNA.}

Nishkakunami España runakunapak punllakunamantami runakunaka supaykuna tukushpa churarikkunakashka yachapayashpa, llakita aychapi apakushkata rikuchishpa shinallatak Curakuna rimakta mana uyanachishpa. Píllaro supaytukushka rurayka, ishki waranka iskun (2009) watapimi,Patrimonio Cultural Inmaterial del Ecuador ukupi yaykushka, kay ruraykunaka sapiyashkami tukuy chiri shalka shinallatak kunuk yunka kullasuyu Abya Yala mamallakta kunapipish, rukunchik Bolivia (Oruro), Venezuela (Yare) Perú (Puno), Revista Vistazopi nishka shinaka (2015).

\section{GUAYAS MARKAPI JUJÁN MOJI- GOSKUNA}

Mojigos tushukkakunaka patsak (100) watakunatami charin, maypimi supay tukushkakunapish chaypitak kan, ashtawanpi- sh paymi ñawpashka tushushpa rikka kan. Rinkunami Alfredo Baquerizo Moreno (Juján) kitipak ñan ukukunata, apyupi tiyarishka; puka churanata churarishka, umapika hatun kachukunayuk shinallatak chupayukmi kan. Kay raymikunaka shamushkami Colonia punchakunamanta kunuk yunka llaktakunapika may sumak kashkata rikuchishpa. Diario El Tiempo (Libro recoge tradicional baile de los Mojigos 2018), rikuchinmi kashna tushukkunaka rikurinmi shuk kutin watapi, chunka iskun shinallatik ishki chunka chusku punllakunapi karwa killapi. Espinosa parlanmi kay ruraykunaka shamushkami waranka - iskun patsak - kimsa chunka (1930) watakunamanta, kay watakunapimi wiñachik José Domingo Delgado rurashka punta Mojigos tushuytaka (La danza de los mojigos 2018).

Fuente: Encalada E. (30 de diciembre del 2017).

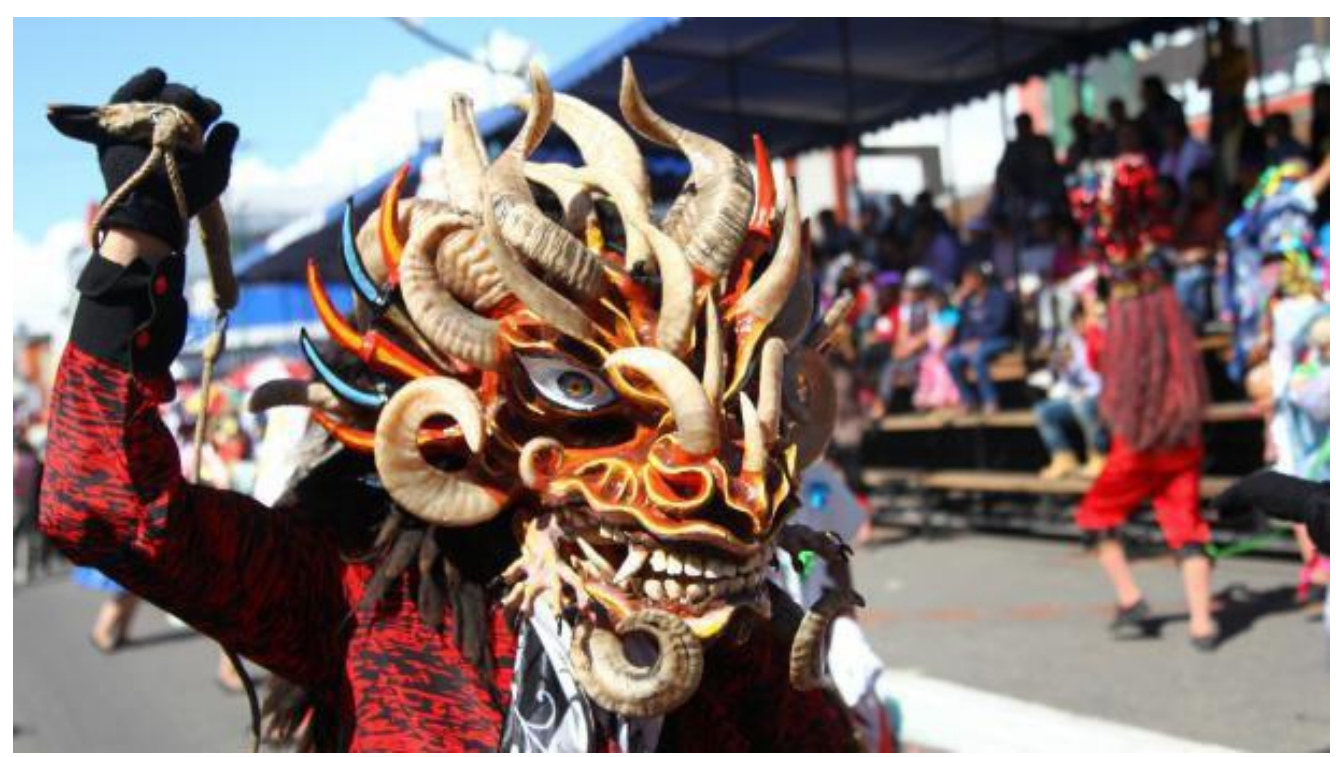

Shuyu 2. Máscara de la Diablada Pillareña (Píllaro, Tungurahua). 


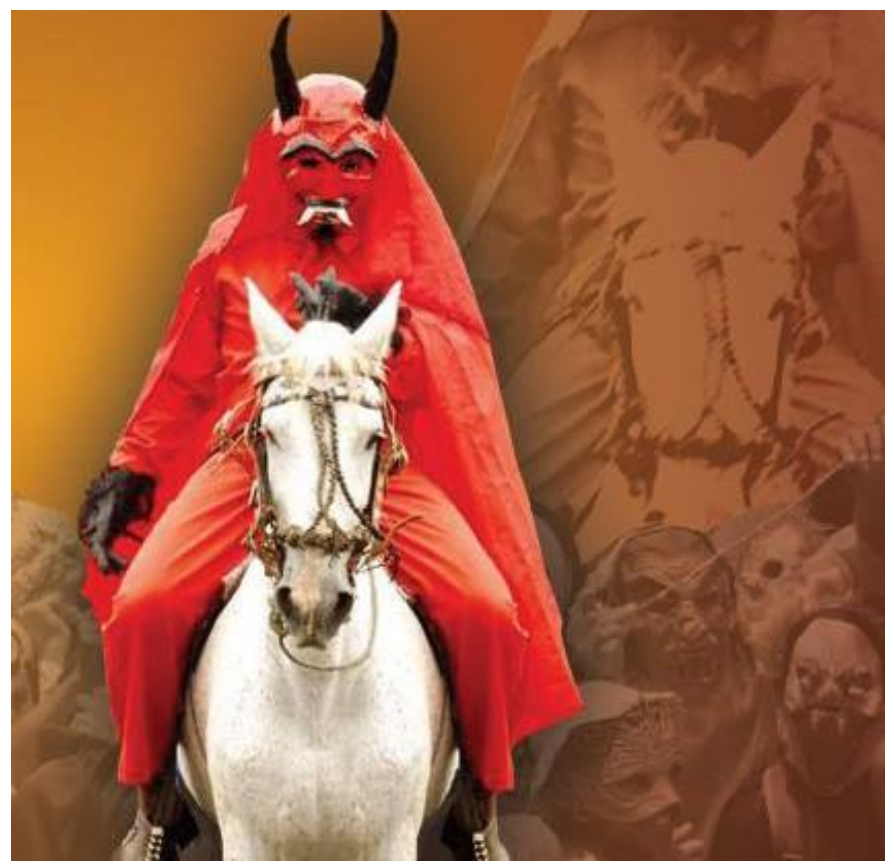

Shuyu 3. Diablo de Los Mojigos de Juján (Alfredo Baquerizo Moreno, Guayas).

\section{PICHINCHA MARKA, CAYAMBE AYA UMA SUPAYKUNA.}

Diablo Huma, ashtawan riksishka diabluma, rikurishkami raymi killapi intipak raymipi (Inti Raymi), hatun sumak raymimi kan ayllu llakta runakunapakka, chaymantami kitipak katunapampapi yaykushpa tushunaman rinkuna.

Aya Huma tukushka rumani kan tukuymanta rikunalla kay chinchasuyu Ecuador raymikunapika, paykunapak willayka kanmi hatun yuyayta charik kashkata rikuchispa, chaymantami llaktamanka chayankuna asishkalla, sumak tullpuykunawan churarishka, kuyak shunkuwan (Cabrera 2018).

Paykunapak ñawikaraka rikuchinmi pachaka ishkay shina kashkata: ñawpa-shamuk pacha, puncha-tuta, hawa-uray, chinchasuyu-kullasuyu. Chunka ishkay akchakunaka rikuchinmi amaruta, haylli yuyaykunata charishkata (Ministerio de Cultura y Patrimonio 2015). 
Fuente: Museo Intiñan (2019).

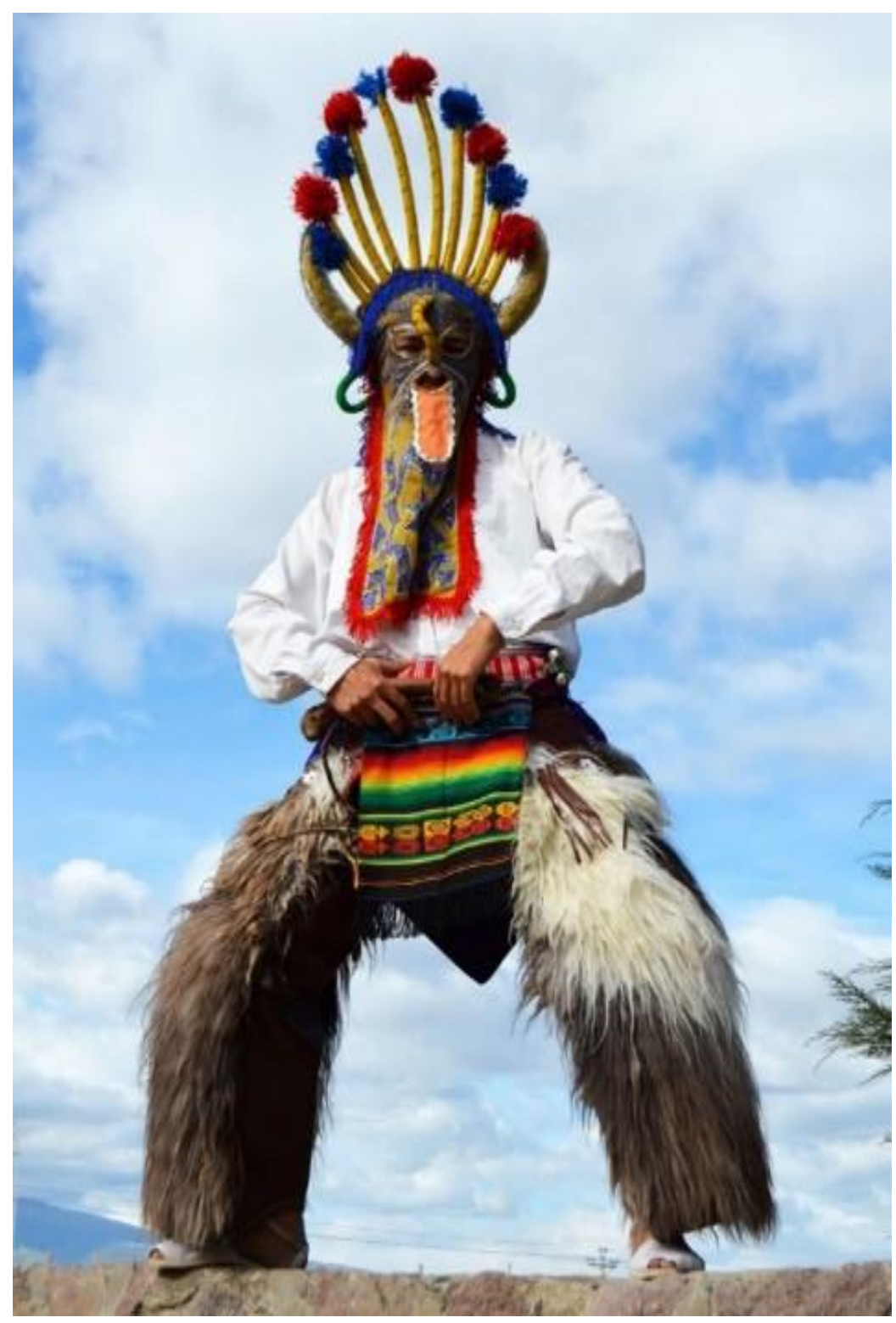

Shuyu 4. Diablo Aya Huma (Cayambe, Pichincha).

\section{PICHINCHA MARKAALANGASÍ SU- PAYKUNA.}

Alangasí supaykunaka. Rikurinmi Semana Santapi, paykunaka Diusta yuyarina wasikunamanmi maypishkachun misata rurakunmi yaykunkuna Viernes Santupi, Sábado de Gloria shinallatak Domingo de Resurreccionpi; rikuchinmi imashina supay Jesuspak kuchupi chashkata manarak chakatarikpi. Kay ruraykunaka ñami charin patsak pichka chunka (150) watakunata. Churarinkunami suninikuk puka churanata, umapipish hatun kachukunawan rurashkata, makikunapika millaypak shiwikunata (Merizalde 15 de abril del 2017).

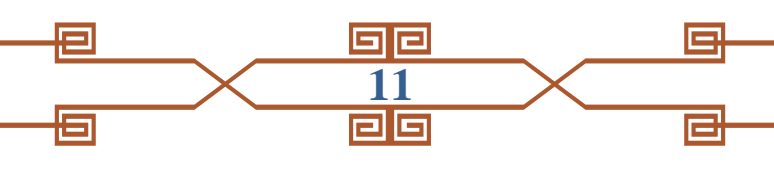


Fuente: Merizalde (15 de abril de 2017).

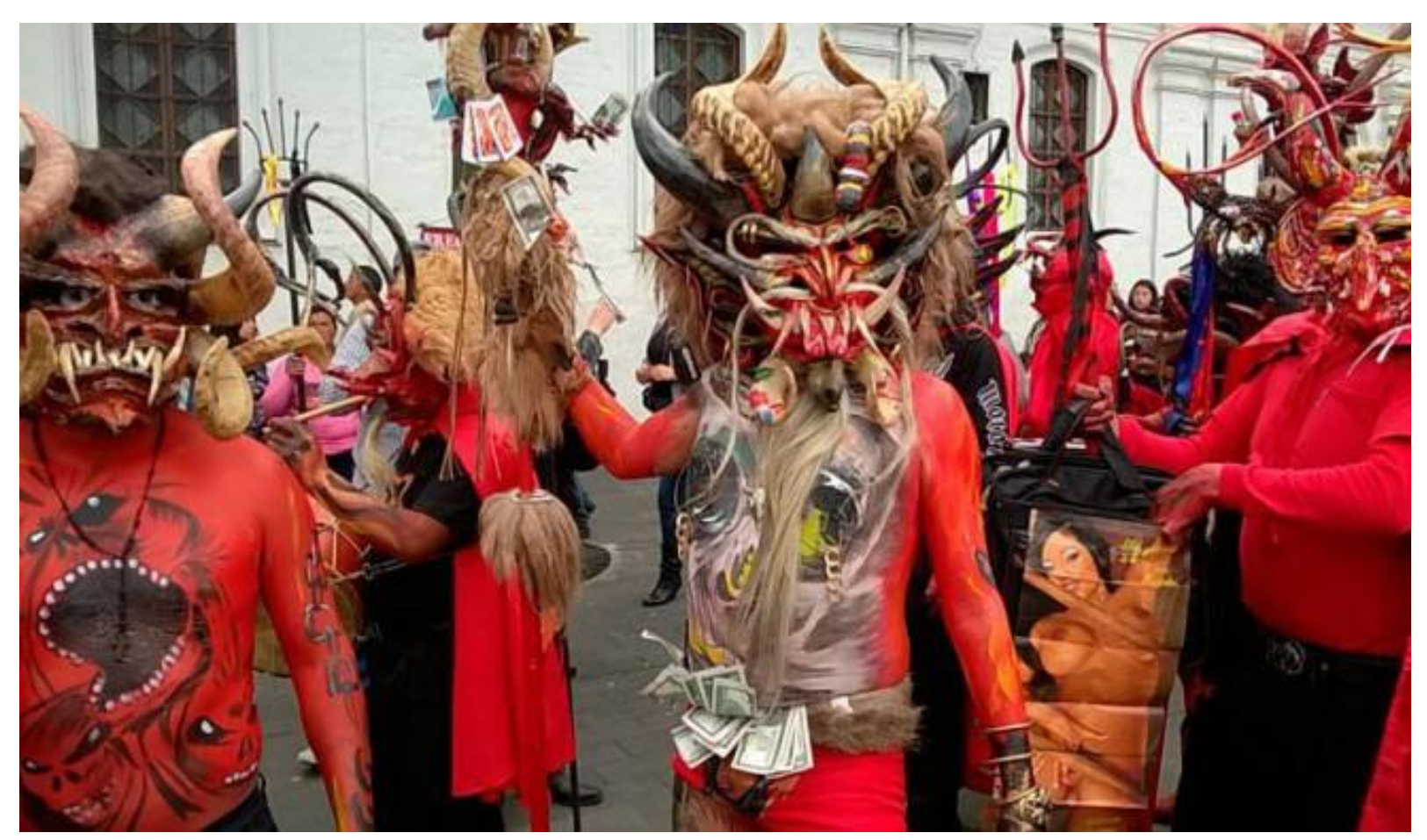

Shuyu 5. Diablo de Alangasí (Alangasí, Pichincha).

\section{SHUKTAK MAMALLAKTAPAK SU- PAYKUNA}

Supay tukushkakunaka rikuchinmi imashina kawsakushkamanta, pushakushkamantapish, ashtawanpish shuktak shuktak rikuchikunawanka rikuchinmi ima shina rayminapi paktakushkata, pawka raymikunapi mana tiyashpapish, shuktak raymikunapimi paktan rikuchikunawan. Mana imata rikushpa ni piwan mana chimpapurashpa, ruraykunapipish ni tullpuykunapipish. Ashtawanpish ishkita rurashpami pushan.

Tiyanmi tukuy shina ruraykunatantachikkunapish, chashnaka shuktak llaktapi ruraykunatapish riksinkapak; kaykunami rikuchin ayllu kawsaypika mana imapish sapalla kashkata, shinallatak ima muhunkunapish mana tiyashkata mamallaktakuna purapika. Hatun rikuyta charinchik supaykunamanta rikuchita chiri shalka llaktakunapi, shinaka charinmi rikunalla churaykunata, kaykunami kan: chupa, suni kachukuna, kurkuyashka sumay rikunalla churanakuna.

Imashinami ñawpapan rikuchirkani, supay tukushka tushukkunaka rikurinmi Centroamérica shinallatak Sudaméricapi Españolkuna shamushkamanta; ashtawanka Catalánpimi wiñarishka, kay supay rikuchika, shamushkami Edad Media pachakunamanta.

\section{PUNO, PERÚ MAMALLAKYA UNE- ÑAPAK SUPAYKUNA.}

Kay supaykunaka kanmi Virgen de la Candelaria raymikunapi tushukkunapak, charinmi ñawpakunamanta sapiyashka ruraykunamanta; kaywanka rikuchinmi runakunapak kawsaymanta (Cosmovisión Andina), ashtawankarin rikuchinmi kaparita allí mana allí ruraykuna chakrurishkamanta runapak kawsaywan mishukunapak kawsaywan.

Yachay chayashkami kay tushuykuna Españolkuna Virreinato Peruman chayachiskata, shinaka cristianismo chayakpika paykuna- 
pak crishkata runakunaman yachachinkapak, chaypimi wacharin allí - mana allí yuyayka, chaypakka misionerukunami chayamushpa kanchis mana allíkunata yachachishka, shinalltak angelkuna supaykunapak hawapi mishashkatapish, paykunapak takikunawan tushuywanpish tantachishpa (Palao 2010).

Hatun Kapak nishkami rikuchin supaykunapak pushak chashkataka, chaymantami shutichiskakuna kapak llakichik awkakunapak pushak kashkamanta, paypak churarishkami kan tukuykunapak yalli rikunalla ñawipi churarishkapish, rikuchinmi kuriwan kullkiwan rurashka shina, shinallatak hampatu rinrin, ashku kirukunashina, hatun kachukunayuk amaruta shina.

Fuente: Palao (2010)

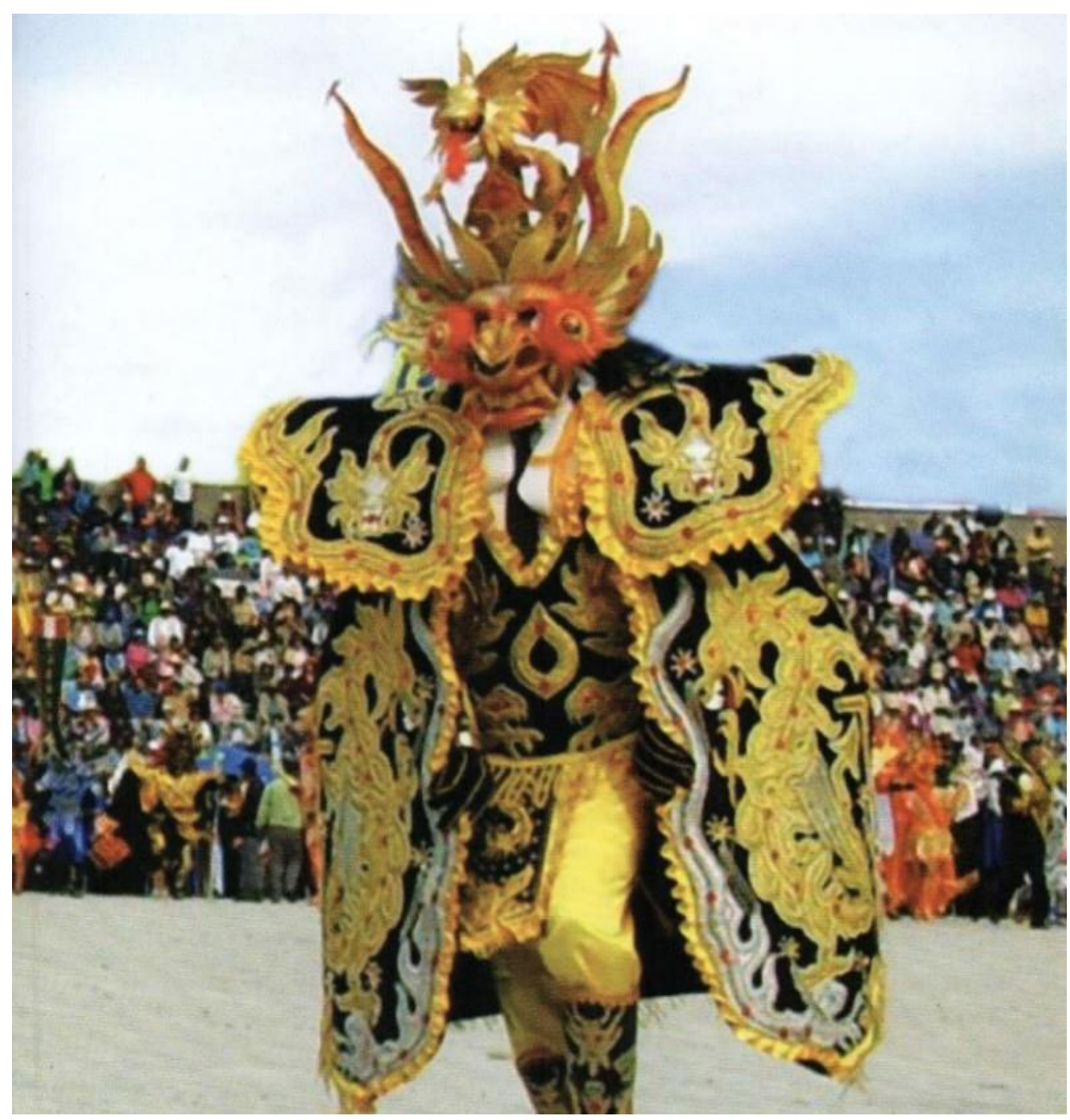

Shuyu 6. Caporal Mayor representante de la Diablada Puneña (Puno, Perú).

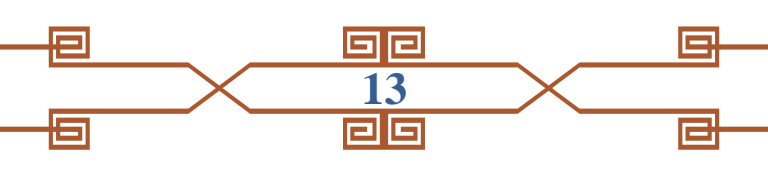




\section{LAMBAYEQUE MARKA, PERÚ MA- MALLAKTAT ÚCUME SUPAYKUNA.}

Lambayeque marka Túcome supaykunaka rikuchinmi kanchis hatun mana allíkunata, rikurinmi Virgen Purisima Concepcion purinapi, panchi killapi. Paykunapak churayka kanmi shuk hatun yana pachallina, pichupika wirpukunata warkuchishka, kunkurikamalla wara, rikra urikunapika cascabelkunata warkuchishka, ushutakunaka espuelasku- nawan, akchaka millmamanta rurashka kinsapi chimpashka; ashtawan supaykunamanta rikunallaka kanmi paypak ñawipi antawan rurashka yanawan tullpushka shinallatak sumakta allichiskakunata, kay ñawi karakunaka rikuchikunmi tukuylaya wiwakunata.

Fuente: Kikin ruray (2019)

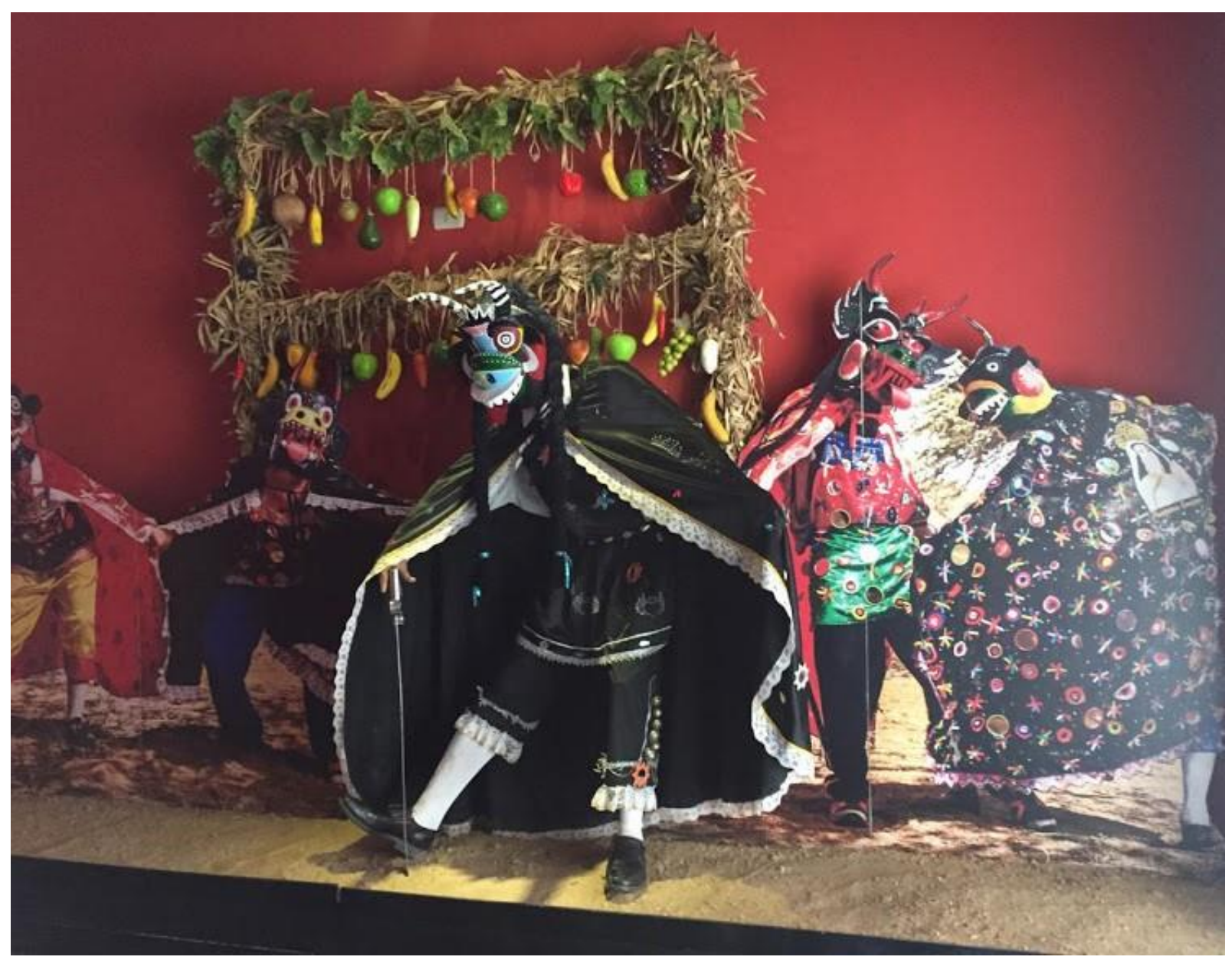

Shuyu 7. Diablicos de Túcume (Ecosmuseo de Túcume, Perú).

回 $>\frac{\text { 回回 }}{14}>$ 回




\section{BOLIVIA}

\section{MAMALLAKTA ORURO SUPAYKU- NA}

Supaymanta carnaval raymitaka Oruro llaktapika willarishka ishki waranka shuk (2001) watapi Patrimonio Intangible de la Humanidad UNESCOMANTA, rikuchinmi kaparita allí - mana allimanta; kati kaparika kanmi Arcangel Miguel kanchis sumak rikuchikunawan, Lucifer supay kanchis hatun llakichikkunawan chimpapurashkata.

Mishukunapak charik yuyaykunapi-sh ñawi churarishkakunapimi riku- rin. Kaywanka charik supaykashkata, paypak charishkakunata kukpish kashkata rikuchinkapak. Makipi churarinapish allitatak rurashkami kan, allitak tullpushka rikunalla piskitakunawan (pañuelo). Ñawikaratapish kashnami rurankuna, kachukunayuk, hampatu, palu, amaru wiwakunaman rikchakta chayka ashka mancharinami kan, shinami rikuchinkuna, kay Uru-Chipaya shinallatak Aymara llaktakunapi. Paykunaka crinkunami Huari Apunchikmi amarukunata kachashka Uro llaktata tukuchichun nishpa (Manuel \& Sejas 2009).

\section{VENEZUELA}

\section{MAMALLAKTA YARE TUSHUK SU- PAYKUNA (SAN FRANCISCO DE YARE)}

Venezuela shinallatik Ecuador mamallaktakunapika, tawka supaykunami llaktakunapika tiyan, ñawpakunamanta rikurishpa shamukka kanmi Corpus Christi de Yare tushuk supaykunawan, chaymantami ishki waranka chunka ishki (2012) watapika Patromonio Inmaterial de la humanidad UNESCO riksik chayachishka (CDCVE 2019).

Kay supay tukushkakunata rikurishkami Corpus misa punchapi mana tawka misaman riskamanta, ñami yallishka chusku patsak (400) watakunata, chaymantami sacerdotika mana Corpus procisionta ruraytukushka, Diosta yuyarina wasi punkupi shayarishpaka kashna nishka: "Mana pi crikkuna Santisimuta apanapak tiyakpika, supaykuna shamuchun" chayta uyashpaka tawkata mancharishkakuna, ñankunapispish ni pi mana rikurishkachu, chashkukpi- mi supay tukushkakunaka hawata hawata tushushpa shamushkakuna Diosta yuyarina wasi punkupi tushunkapak, pauykunaka mana yaykuy tukushkakunachu. Supaykunaka churarinkunami puka churanakunata, ramuswan rurashka Rosariota kunkapi warkuchinkuna, waranka iskun patsak chusku chunka pusak (1948) watakunamantami shuktaklaya churaykunawan shamushka, yurak warakuna hawapika tullpushkakuna amarukunata, kiwakuna, inti, killa tullpuykunata makiwan rurashpa, shinallatak puka yurak sunikuk chakillikunatami churankuna. Shinallatik churankunami escapularioskunata, ramuswan rurashka chakanakunata, wallkakunatapish. Ñawikaraka allpawanmi rurashka kan, pankakunawan, trigu kutawan shinallatish tullpuykunawanpish, supay kashkatatakmi rikuchinkuna, tullpunkunami pukawan kashpa mana kashpaka yanawanpish. Kirukunapish hatun kanipaklla, washamanka apankunami puka hatallita chayllatakmi ñawita tapallinapakpish kan (Ramírez 2018). 


\section{YACHAYÑAN}

"Kay taripaykunaka shuk ñantami katin, chawanmi yachaykunaman chayanchik ima yuyayta charishkata willankapak, hamuktarinkapak, shinallatak mushukrarinkapak, shinallatak ñukanchik runakunapak kawsayta punchanta allichishpa rinkapak" (Monje 2011).

Llankaypi taripashkakunata paktankami imallata rikushkata yachayñanpi katishpa, chaymantami katinka yachayñan llankaypak nishkashina: etnografía, antropología shinallatik parlanakushka kunawan, chayka rikichinmi aylluyarinata pactankapak yuyaykunata taripashpa. Ashtawanka parlashkushkakunawanka riksik chayarinmi kawsaymanta, yuyaykunamanta, muskuykunamantapish (Díaz-Bravo, Torruco-García, Martínez-Hernández \& Varela-Ruiz 2013).

Kay yachayñan llankayka charirinmi etnografía rikushkapi "Charirinmi paktana yuyaykunapi ima ruraykunapish, paktaykunapish, chaymantami kawsaypika shunkuyashpa asha asha rin, chaywanka yuyachitukunchik sapalla kawsakkunapapish ayllupi kawsachun, shinaka yuyaykunata ayllukuna ukupi sinchiyachinapak" (Rodríguez, Vera \& Vargas 2011:30).

\section{PAKTASHKAKUNA - RIMANAKUYKUKA}

Centroamérica shinallatak Sudamérica mamallakta kunapika sumak rikuchikuna, raymikuna, tushuykunami wiñarishka manarik Españolkuna chayamukpi, ñawka yuyakkunapak sumak sakishkakunawan, Españolkuna chayamushka washaka ashkatami allichirishka, chaywanka tantallakana kashkata rikuchispa.

Ecuadormamallaktapikatawka raymikunami tiyashka ñawpa yuyakkunapak pachamanta, kay raymikunaka sinchiyashpami shamushka tawka ruraykunawan. Españolkuna chayamushpaka paykunapak yuyaykunatami chakruchishka, chaymantami shukllapi tantarishka runakupak ruraykunawan mishukunapak ruraykunawan.

Wawa puri raymi Riobamba kitipika hatun raymikunapak ukupimi kan, Chimborazo marka ukupika chusku patsak Wawa puri raymikunami paktan, kamay - kulla killakunapi. Ishki waranka chunka pusak (2018) wata, kuski killa chunka punllapimi, Patrimonio Cultural Inmaterial del Ecuador riksichishpa churashka kay sumak raymitata, chaymantami chay punchaka hatun raymita rurashakakuna Chimborazo markapi kawsakkunawan, tushukkunawan shinallatak kay raymita watanta rurakkunawanpish.

Kanchis riksishka churarishka runakunami rikurin kay raymikunapika, paykunataka churarishkapimiriksinallakan.Ashtawanpish rukuchinmi shukllapi tantanakushkata mishukunapak ruraykunawan, kay churarishkakunami rikurin kurikinkikuna, sacha runakuna, tushukkuna, asichkukuna, ashkukuna, kushillukuna, ashtawankaSupay anta tukushkakuna.

\section{SUPAY ANTAPAK RIKURI}

Supay antaka rikurishkami Wawa Kapak Kapakkunapak puri raymikunapi Riobamba kitipi ñawpa watakunamanta, Ángel Valdivieso parlashpaka ninmi (Diablos de Lata, una tradición riobambeña 2017), barrio Santa Rosapimi rikurishka chaymantami apan tsawar puchkata kimsapi chimpashkata. Yachachik ojalateroskunami allichishka ñawi karatapish cartonmantaka antawan rurashpa.

Tawka antropologokunami killkan imashina shuk runa supay tukushkata. Ninkunami kaykunaka shamushkami karumanta purik runakunamanta, chaymantami supay tukushkakunaka mana paylla tushun, tukuykuna tantanakushpami tushunkuna. Shuktak antropologokunaka tullpunmi supaytaka kachukunayukta, asialta apashkatapish, rikushkanchikmi kinkuna Corpus raymikunapi, tushukkunapak washapi rikukta. Chay supaykunaka makipi 
apanmi rebenque nishkatapish (Vallejo 2012).

Kay taripashkakunaka rikuchinmi Supay Anta imashina kakta, maymanta rikurishkata, ima yuyaywan kaktapish, tushushkawan, churarishkawan, paypak paktaywan rikuchin Wawa Kapak Kapakkunapak puri Riobamba kiti raymikunapi. Shinallatak rikuchinmi runakunapak rikuchiwan Españolkunapak rikuchiwan shukllapi tantanakushkata yallirikushkatapish.

Runakunapak kaysaypika millay supay llakichik yuyayka mana tiyashkachu, ashtawanpish kay paktaykunaka kawsaymanta, murukunata hapishkamanta, inti taytamanta, killa mamamanta, urkunamanta yupaychanami kashka. "Españolkuna chayamushpami paykunapak ruraykunawan chakruchishka, kulla raymi pactaykunawan shinallatik Wawa Jesús wachari raymiwan shukllata rurashpa." (Á.
Valdivieso, kikin willay, 04 de enero, 2019).

\section{SUPAY ANTA MAYPI KALLARISHKAMANTA}

Supay antaka shamushkami runakuna supay tukushkata rikushkamanta, ashka watakuna washamanka ñawi karapish cartonwan rurashka, yurak churarishka, kunkapika taswar puchkawan watarishkami kashka; ñanta achikchishpa rinkapakka faro niskatami apakkuna kashka, chay pachapika mana kunan shina achik tiyashkamanta. "Supay tukushka rurakunaka Chimborazo marka Cacha kitilli raymikunapimi rikuchik kashka." (Á. Valdivieso, kikin willay, 04 de enero, 2019).

Supay Anta tukushka runami may sumak rikunalla kan paypak churarishkawan tushushkawanpish. (Á. Valdivieso, kikin willay, 04 de enero, 2019). Eduardo Yumisaca (kikin willay, 03 de enero, 2019) supay Sesquecentenario tukushkunapak

Fuente: El diablo de hojalata participa en "El Pase del Niño" (26 de enero de 2017).

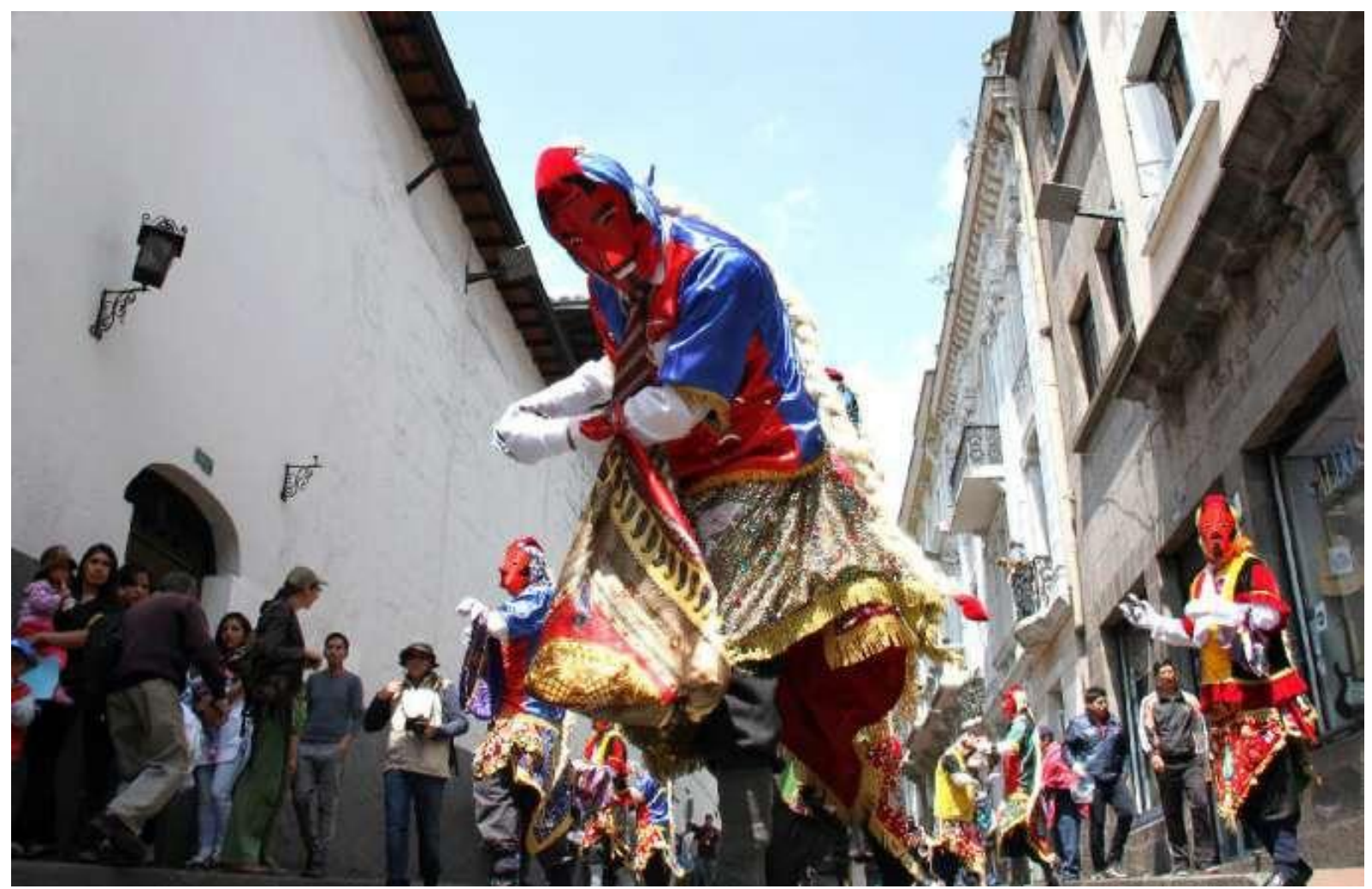

Shuyu 8. Diablo de Lata en el Pase del Niño Rey de Reyes (Riobamba, Chimborazo). 
pushak shinallatik Danny Fuenmayor (kikin willay, 06 de enero, 2019), Santos Supay tantanakushka ukupi wankurik, ninkunami kay supak tukushkakunaka Cachapimi wacharishka, chay punchakunapika Riobamba kitipak ayllullaktallami kashka, paykunaka Yaruquista uriyashka chayakkuna kashka Santa Rosaman, chapimi hojalateroskuna tupakkunakashka; shina kakpitak Santa Rosapitak Wawa Kapak Kapakkunapak Diusta mañana tiyarishka, "chaymantami Wawa Kapak Kpapakkunapak purinaka kallarishka, ashtawan ñawpakunamanka Kapak Magukunapak raymikunatami rurakkuna kashka, Españapi raymikuna rurarikshina."

Alex Proaño parlanmi, Cachamanta Supaykunaka cartonmanta rurashka ñawikaratami churakkunakashka ninmi, Santa Rosaman Chayamukpiki ñawikarata rurakkunaka antamanta rurashkakuna.

\section{UNANCHA}

"Supay Anta rikuchika mana ima millayman shutikutakuna yuyaytata rikuchinchu" (Á. Valdivieso, kikin willay, 04 de enero, 2019). Daniel Fuenmayor (kikin willay, 06 de enero, 2019) rikuchinmi, kay supay tukushkallami mana Españolkuna llakishishkallamanta wiñarishkata rikuchin, chaymantami colonizadores catolicokunaka chaypi charirishpa hawalla tantachishka procesionkunapi mana allí tiyashkata rikuchinkapak, "kayta mana kunkarinakanchik, runakunapak yuyaypika mana allí católico yuyayka mana tiyanchu, ashtawanpish tukuy raimikunami charina kan allita mana allita, ishkita rurashpa yallinapak, chaywanka runakunapak kawsayta chimpapurankapak." Lajo yachakka ninmi (2005), kay pachapi imalla tiyashkaka runakunapak rikuypika ishkitakmi kan, tukuy ima tiyashkapish ishkimi kan, chaymantami ishkipak ukupipish allipish mana allipish tiyan ninkunami.

\section{SUPAY ANTAPAK TUSHUY}

"Manarak tushushpa, Supay Antmantaka, yachanami kanchik pipak shinallatak imapak tushukta" (Á. Valdivieso, kikin willay, 04 de enero, 2019). Valdivieso, kay hawaka ninmi "rikuchi tushuymi kan mana yanka tushuykunashina"; charinmi paykunapaklla tonada nishka takita, "supaypak tonada" ñawpakunamanka tambur pinkullupimi takikkunakashka kimsa pachakunata rurashpa; shinallatak shalak shalakkunawanpish, kay takinawanki kayakkuna kashka, kunpidankuna sumay tunukunakushpa supaykuna tushuchun, yuyarina kanchik kimsa pachakunata ruranata tushunapak, alliman llukiman. Chaytami ninkuha punteado nishpa.

A. Valdivieso (kikin willay, 04 de enero, 2019) y Gonzalo Carrasco (kikin willay, 06 de enero, 2019) parlashpaka ninkunami, kanchish watakunatami supaytukushpa tushuna kan, kay kanchish watakunapika chusku shinami tiyan, kaykunami kan: wamra, musu, runa, yuyak. Kanchis watakuna kipa chay shinalla tushushpa katishpika shutichinkunami "Supay supaykunapak" mana kashpaka "supaykunapak apu".

Watantami rikuchinkuna hunkay shuk punchapi, shinallatak watantami shuktakshina rikuchikunata rikuchinkuna:

shukniki watapika tushunkuna uma ñakcharinawan - shalak shapakwanpish, ishkayniki watapika nina hapichinawan - shalak shalakwan, kimsaniki watapika hayak yaku purpuwan - shalak shalakwan, chuskuniki watapika asialwan - shalak shalakwan, pishkaniki watapika wawata rurashkawan - shalak shalakwan, suktaniki watapika shutichinkunami "wakay siki supay anta" kanchisniki watapika ña anchurinallami kashka. Kanchishniki watapika tushunkunami farolwan, chay ukupika shuk yurak urpita churashpa, chayka rikuchinmi Diospak Sumak Samayta, misa tukurishka washa mana kashpaka tukuy tushushka washami kacharinkuna, faroltaka ninawan hapichinkuna urpitaka hatarichispa 
kachan, kaywanka rikuchinki (significado) Wawa Jesusman ñukanchik samayta aychata kukushkata. (Á. Valdivieso, kikin willay, 04 de enero, 2019).

Kay tushukwanka rikuchinmi Wawa Kapak Kapakkunpak puri hatun kashkata, maypimi Supay Antaka mañaykunata churan, hatunyachinpish Wawa Diusta" (B. Guzmán, kikin willay, 06 enero, 2019). "Kanchis watakunata tushuyka kanmi shuk paktachi, kunpiday, aylluyari Wawa Kapak Kapakkunawan" (J. Santos, kikin willay, 06 de enero, 2019).

Kanchis yupayka kanmi paktashkatak, kanchis punchakunaka kanmi Yaya Apunchik kay pachata rurashkamanta yupaychay, kanchis watakunaka mana allí rurashkakunamanta yuyarishpa allichirina, chaymantami kanchis watakunata tushuna kan. (X. Villagrán, kikin willay, 06 de enero, 2019).

Jhony Orozco shinallatak Byron Guzmán, taxi antawata purichikkuna, kashanmi ninkuna, "tiyanmi kashna nikkuna, mayhan mana kanchis watata tushushpa paktachikta, supaymi apashpa rin nishpa". Juan José Fuenmayor (kikin willay, 06 de enero, 2019), los Santos Diablos ukupi wankurin, ninmi "watakunaka nishaninmi Supaypak wishina, maypimi rupanaman chayana kan". Danny Fuenmayor (kikin willay, 06 de enero, 2019), ninmi "kanchis watakunata tushunaka rikuchinmi kanchis mamapachapi runakunapak kawsaypi yallishkata yupaychashpa: yaku, wayra, allpa, inti, killa, kuyllurkunata.

"Kanchis watakunata tushushpa tukuchishpaka llakikunamantami kishpirin,

Fuente: Kikin ruray (2019)

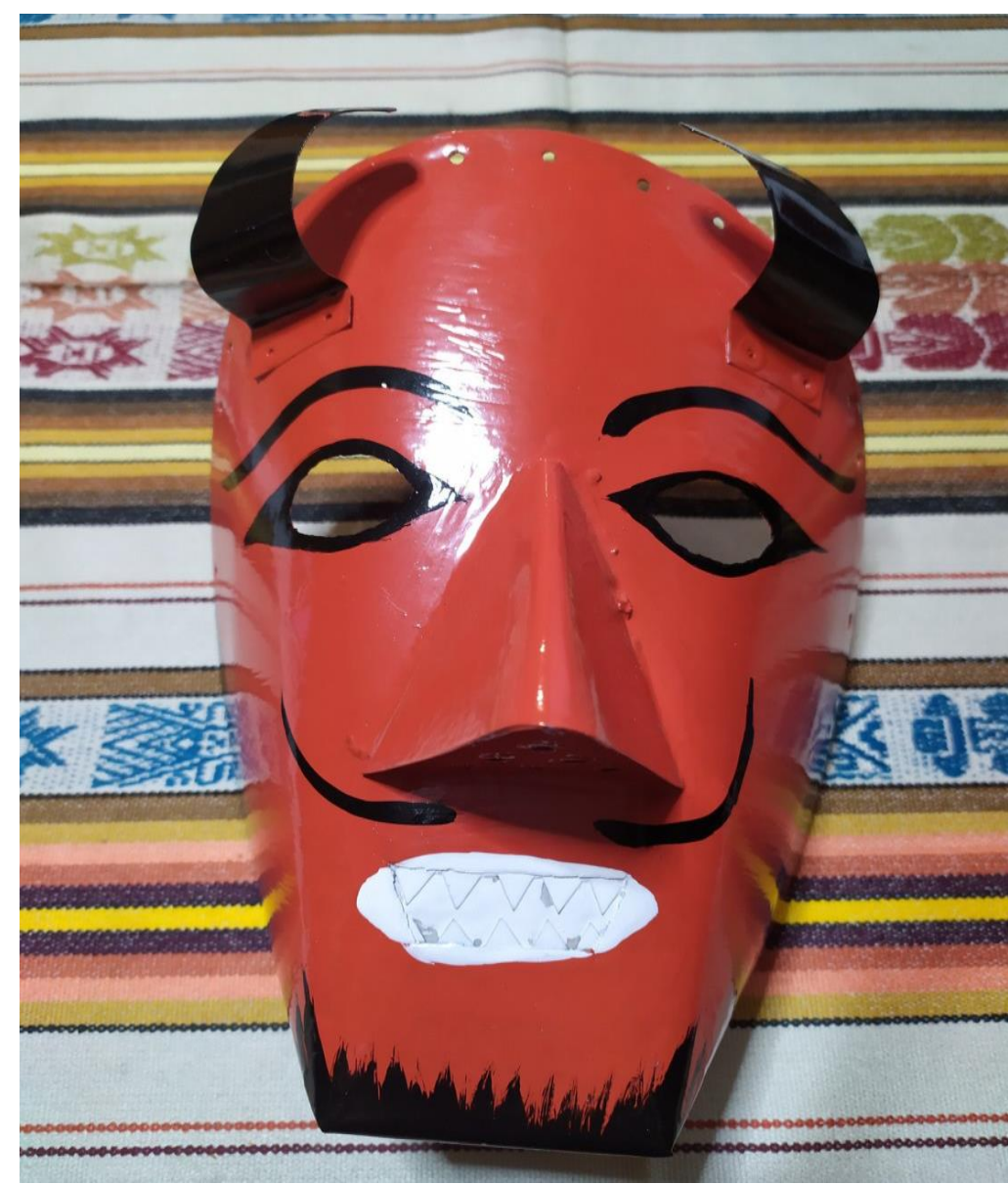

Shuyu 9. Careta Básica del Diablo de Lata color rojo y negro.

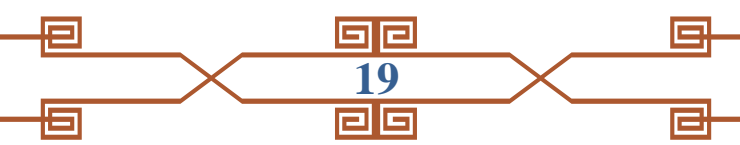


shinaka charitukunmi hawa pachaman yaykunata" (D. Tuquinga, kikin willay, 06 de enero 2019).

\section{SUPAY ANTAPAK CHURAYKUNA}

Supay Anta tukushkami may sumak rikunalla kan, kay Wawa Kapak Kapakkunapak purikunapika, churarinkunami yana charol ushutata, shinallatak yana warata, yurak ruwakata, sumay kunkapi warkuchinata, suni ruwakata ankaswan pukawan rurashkata, yurak makipi churarinata, kunawan, piskitawan chimpashka, ñawikara, shalak shalakwanpish. (A. Valdivieso, kikin willay, 04 de enero, 2019).

Kay supaykunaka charinmi shuktak shuktak wichiyaykunata, shukniki watapi tushushpaka churarinkunami yanawan pukawan tullpushpa ñawikarata shinallatak ankaswan pukawan churana; watakuna yallikpika ashawan ashawan may sumakta allichirinkuna tukuy churaykunapi; chaywanka Supay Hatun Apu tukunkakama, kaykunapimi riksitukurin ñawi karapi chitata charikta rikushpa, shinallatak paypak churanapi sumakta allíchishkata rikushpa. (D. Tuquinga y E. Yumisaca, kikin willay, 06 de enero, 2019).

\section{SUPAY ANTAPAK ÑAWIKARA}

"Shukniki ñawikaraka rikurishkami Cacha kitillipi, cartonwan rurashka chaypi kawsak runakunamanta" (B. Guzmán, kikin willay, 06 de enero, 2019).

José López (kikin willay, 05 de enero, 2019), Hatun Tushukkunapurapi wankurishka, ninmi Supay Antapak ñawi kara rikuchika

Fuente: Kikin ruray (5 de enero de 2019).

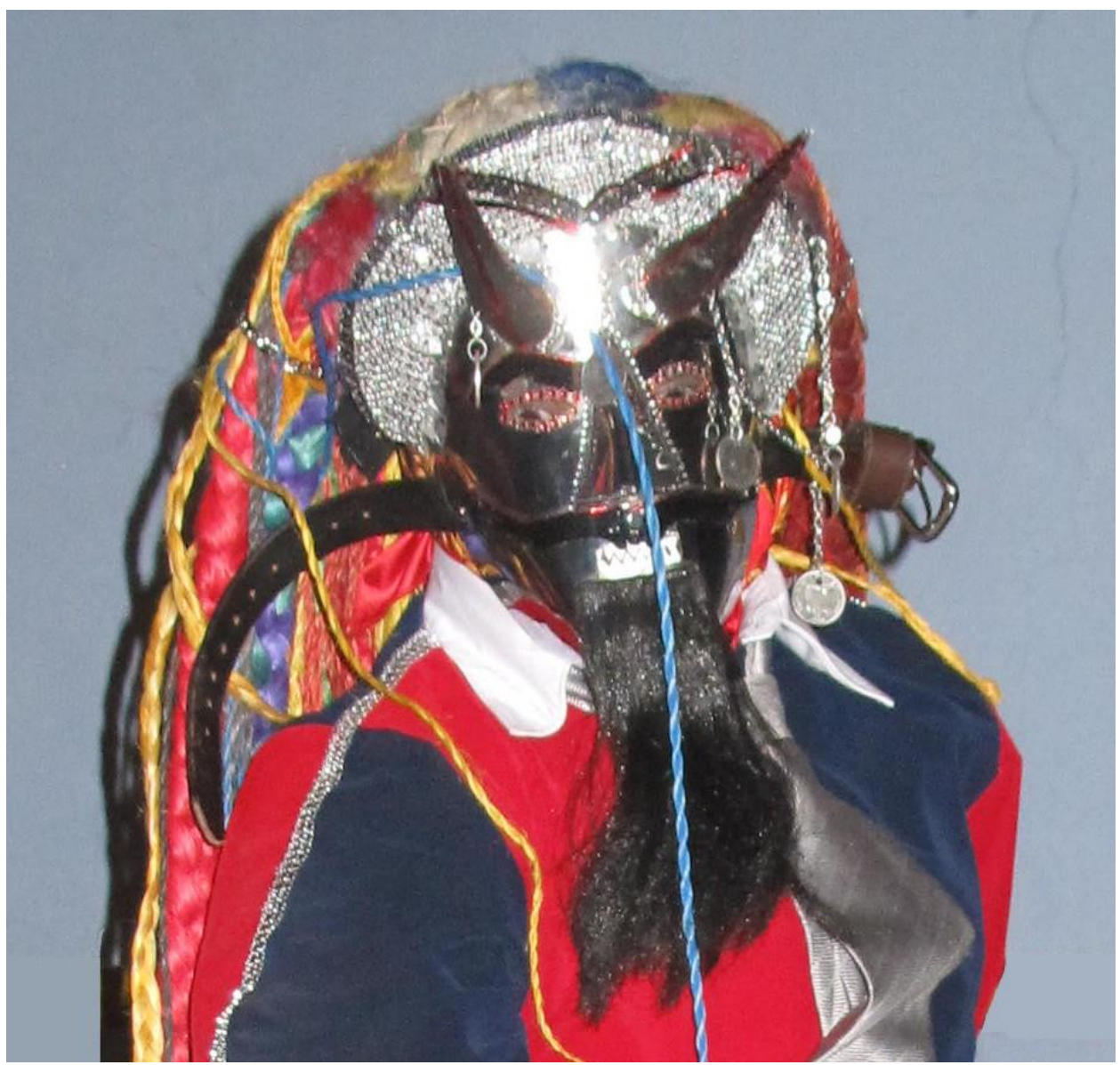

Shuyu 10. Careta del Diablo Mayor o Diablo de Diablos.

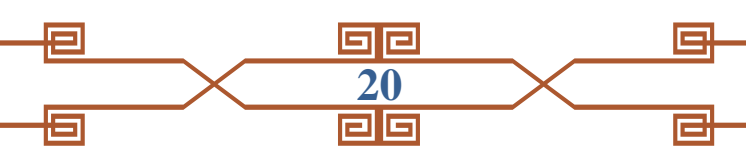


kanmi shuklla tukuy Abya Yaya kullasuyu llaktakunapika, kay shina rikunaka mana may shuktak mamallaktakunapika tiyanchu, tiyanmi Ecuador mamallaktallapi kaypimi wiñarishka ñawikaraka antawan rurashka yanawan pukawan shinallatak tawka watakunata supay anta tushushpa shamushkamanta, ashkata allichishkaka kanmi ñawikara, yana chitata charinkakama.

\section{TSAWAR CHINPA}

"Tsawar chimpaka rikuchinmi supaypak akchata, watanta tushushpa rikpika akchaka wiñachishpami rin ashpapi chayankakama" (J. López, kikin willay, 05 de enero 2019). Runakunapak yuyaypika chimpaka hamuktachinmi waka kashkata mamapachawan tantalla kashkamanta. Runakunapak kawsaypika, akchaka kanmi shuk sumak kurishina, "kaysayta kuk kashkamanta. Mayhan ashtawan suni akchata charikka charitukunmi alli willanakuyta pay maypimi kawsakunpi" (Alvarado 2016). "Allpapi chinpa chayakpika, Pachamamaka ashtawan paypak sumay samaytami kun tushushpa katichun" (X. Villagrán, kikin willay, 06 de enero, 2019)

\section{PISKITA}

Wiksa uranikpika churankunami ishki piskitakunata, shuktaka ñawpakman kayshuktaka washaman, kay piskitakunaka apanmi chakana, chunukari rikuykunata, wirpukunatapish, caliztapish, mana kashpaka Wawa Kapak Kapakkunapak shuyuta apankuna, shuktak punchakunapika mana imata apankunachu. "kimsaniki piskitataka umapimi watarinkuna ñawikarata churarikpika ama chukrichichun" (J. López, kikin willay, 05 de enero, 2019). "kimsa piskitakunaka rikuchinmi Calpi, Cacha, Yaruquies kitillikunata" (K. Altamirano, kikin willay, 06 de enero, 2019).

\section{TULLPUYKUNA}

“Ankas puka tullpuykunapak ñawpakmanka kay rikuchik tullpuykunami tiyashka, yurakka allita rikuchik kutin yanawan pukawanka mana allita rikuchik católico crikkunapakka, chay kipami hapishkakuna kitipak tullpuykunata Supay antata churachinkapak" (X. Villagran, kikin willay, 06 de enero 2019).

"Supay Antakunaka shuktak shuktak tullpushka churana kunatami churarishpa rinkuna, puka shinalltak ankas tullpuykunami Chimborazo Supaykuna kashkata rikuchin" (J. López, kikin willay, 05 de enero, 2019).

J. Fuenmayor (kikin willay, 06 de enero, 2019) yachakka ninmi "kay tullpuykunaka rikuchinmi Riobambapak yugu yawarta."

\section{SHALAK SHALAK}

Ángel Valdivieso kaytami nin, shalak shalak tukanaka kaparishkatami uyachin, ñawpakunapika mana kunan punchakunashina willachik kayachikkuna tiyakpika, sinchitami kaparikkuna kashka, chaymantami wiñachishkakuna shalak shalak takinata kumpidashpa kayankapak". Ashtawankarin kanmi shuklla rikuchi wawa puri raymika, paypaklla tunutapish charin, tukuy tushukkunatimi kimsa pachapi tushuchispa pushashpa rinkuna (kikin willay, 04 de enero de, 2019).

X. Villagrán parlashpaka ninmi, shalak shalakwanmi sumakta tushunkuna (kikin willay, 06 de enero, 2019). D. Fuenmayor parlashpapish ninmi, "shalak shalakwanmi Supay kaypi kashkata rikuchin" (kikin willay, 06 de enero, 2019).

J. Fuenmayor, Juan Carlos Chávez, Byron Guzman y Paulo Ricaurte, tukuy paykunapish ninmi, shalak shalak kunawanmi rikuchin campanakushka tunukanata shina, kaywanka supaymanta shamushka mana allí yuyaykunata manchachispa kachankapak (kikin willay, 06 de enero, 2019).

Klever Altamirano parlashpaka ninmi, shalak shalakwanka rikuchinmi Riobamba Santa Rosa ojalateros rurakkunata (kikin willay 06 de enero, 2019). 


\section{ACHIKCHIK}

Kay achikchiktaka kanchis watata Supay Anta tukushpa tushukmi apashpa rin, chaywanka riksichinmi ña chay tushukka paypak ari nishkata paktachikushkata. (J. J. Fuenmayor, kikin willay, 06 de enero, 2019). "Chay achikchinapika shuk urpitami apan, riksichinmi Diuspak Sumak Samay chaypi kashkata, ña tukurikpika urpitaka kacharinkuna kutin achikchiktaka tukyachinkuna" (B. Taday, kikin willay, 06 de enero, 2019).

\section{SUPAY ANTAPAK PAKTACHI WAWA PURINAPI}

Valdivieso (kikin willay, 04 de enero, 2019) parlashpaka kashanami nin, "Riksichinmi Supay Anta Wawa Kapak Kapakkunapak purita hatunyachishkata, shinallatak tushushkawan Wawa Jesusta yupaychaktapish".

Paykunami wakichinkuna Wawa Jesupak imagentaka, shinallatak paykuna chaypi kashkawanka manchachinkunami supaymanta shamushka millay yuyaykunata, Supayka mana millay kanchu, ashtawanpish charinkunami shuk paktachikunata sinchi sinchi kanapak.

Shinaka, Supay Antami kan wiñaypak Wawa Kapak Kapakkunapak ayanapak, payta ñawpashpa wakichik, shinallatak Wawapak shuyu purichun ñanta paskakpish. Paypak rikuchimi tikuymanta yalli sumay kan.

Shinallatak J. Orozco (kikin willay, 06 de enero, 2019) riksichinmi, "Supay Anta Wawa puripi mana tiyashpa, ima ruraypish mana tayanmanchu". "Paypak tushushkawanmi mana allikunata anchuchin Wawa Jesusman kuchuyachunkuna. (J. J. Fuenmayor, kikin willay, 06 de enero, 2019).

Supayka tikuy puri raymi paktaykunapimi kan, "paymi kushiyachikun allita Wawa Kapak Kapakkunapak raymi paktachunka, Wawa Jesusmantaka karu karullami kan, shinapish paypak wakichikmi kan" (X. Villagrán, kikin willay, 06 de enero, 2019).
B. Guzmán parlashpaka ninmi, paymi kashka shukniki rikuna Wawa Kapak Kapakkunapak puri raymipika (kikin willay, 06 de enero, 2019). Wawa Jesús kay pachaman chayamushkatami raymiwan paktachin (A. Casco, kikin willay, 06 de enero, 2019).

Kipapika, P. Ricaurte rikuchinmi kayta "Supay Antaka kunmi kushikuyta Wawa Jesús purichun, tukuy allikunapika minishtinchikmi rikunata ima mana allí paktakushkatapish, kaykunapi rikuytukunchik yeng - yang, yurak - yana, chaytami paktachin Supay tukushkakunaka, kushikuyta tukuypi kunapak" (kikin willay, 06 de enero, 2019).

\section{TUKUCHIKUNA}

Kay tukuy taripashka yuyaykunawan uchilla yuyaypi churashpaka ni tukunchikmi, Supay Anta Wawa Kapak Kapakkunapak puri Riobamba raymikunapika, chimpapuranmi shuktak Sudamérica mamallaktakunapi rurashka raymi kunawan; chaymantami shuylaya ruraykuna kan paykunamantaka, shuklaya ñawikarata charishkamanta, Riobamba Santa Rosa hojalateros rurashkamanta. Mana hawalla rikuchipakchu kan, shamunmi ñawpa yuyakkunapak yuyaymanta crishkamantapish, chimpashkapi rikurishpa, tushuypi, shalak shalakkunapi; shinallatak rikunchik Españas. XIV Corpus Christi supay tushukkunamanta shamushkata.

Hamuktarinchikmi colonizadores católicos Abya Yala allpakunaman chayamushkamanta kay supay rikuchikunaka ashtawan sinchiyarishkata (similar al Diablo Huma) chaymantamy chimpapurankuna España raymi supaywan, chaywanka sinchiyachinkapak kunan puncha supay tukushkakunata, chaywanka rikuchinmi tukuymanta yalli Wawa Kapak Kapakkunapak wakaychinata, kaykuna mana tiyakpika raymika mana paktanmanchu.

Riobamba Wawa puri raymipi Supay 
rikuchika mana ima mana allika kanchu, ashtawanpish ashtawanmi aylluyachin Wawa Jesustapish wakichin millaykunamanta, chaymanta Supakta mana rikuytukunchikto millay kashkataka, wakichik allí yuyaykunata wiñachik kashkamanta.

Watakuna yallikpika, tukuy ruraykunami allichirishka, Wawa Jesús puri raymikunapish, churaypish, ñawikarapish, Supaypak tushuykunapish. Chaymantami tawka allí kay tukuy taripaykunaka yuyarinkapak, ñawpa rikuchikunata kunan puncha rikuchikunatapish, Supay ruraykunata mana chinkachinkapak shinallatak shuktak ruraykunata mirachiska ama llakichinkapak, maypika mana riksishkamanta ama chinkarichun.

\section{RIKUSHKA KAMUKUNA}

Alvarado, A. (14 de enero de 2016). La cosmovisión se reflejaenlacabelleradel otavaleño. El Comercio. Recuperado de https://www.elcomercio.com/ tendencias/cosmovision-cabelleraotavaleno-cultura-vestuario.html

Cabrera, J. (2018). Aya Uma: Aportes desde las tradiciones orales a la imagen y el concepto. Revista Nawi, 1(3), 103118.

Centro de la Diversidad Cultura de Venezuela en España. (2019). Los Diablos Danzantes Del Corpus Christi. Recuperado de https://cdeve. es/actualidad/los-diablos-danzantesdel-corpus-christi?fbclid=IwAR0-Ljn u7SUOiL6eBwSA6ZQnxcxxw4Os7o 4ZQeKoByw3eB5jYEwUvgDYpjc

Díaz-Bravo, L., Torruco-García, U., Martínez-Hernández, M. \& VarelaRuiz, M. (2013). La entrevista, recurso flexible y dinámico. Investigación en Educación Médica, 2(7).

Diablos de Lata, una tradición riobambeña. (2 de febrero de 2017). La Prensa. Recuperado de http://www.laprensa. com.ec/interna.asp?id=11881\#.

\section{XSKjNZMzbjA}

Diablos: símbolo de la alegría popular. (2 de septiembre del 2018). El Comercio. Recuperado de https://www. elcomercio.com/tendencias/diablossimbolo-alegria-popular.html

El diablo de hojalata participa en "El Pase del Niño". (26 de enerode 2017). La Hora. Recuperado de https://www. lahora.com.ec/noticia/1102024408/ eldiablo-de-hojalata-participa-en-elpase-del-nio

Encalada E. (30 de diciembre del 2017). La Diablada de Píllaro, un ícono turístico nacional. El Comercio. Recuperado de https://www.elcomercio.com/viajar/ diablada-pillaro-icono-turisticoecuatoriano.html

En Ecuador, cientos de "diablos" expresan su rebeldía contra la represión. (viernes 9 de enero del 2015). Revista Vistazo. Recuperado de https://www.vistazo. $\mathrm{com} / \mathrm{seccion} / \mathrm{pa} \% \mathrm{C} 3 \%$ ADs/en ecuador-cientos-de-diablos-expresansu-rebeld\%C3 \% ADa-contra-laopresi\%C3\%B3n

Espinosa, J. (2018). Historia del Diablo y los Danzantes de Jujan en el Ecuador. Los Mojigos. Ecuador: Espinosa

Irady, B. (2018). Con los Diablos Danzantes de Corpus en Castilla - La Mancha. Venezuela. Recuperado de http://www. diversidadcultural.gob.ve/actualidad/ con-los-diablos-danzantes-de-corpusen-castilla-la-mancha

La danza de los mojigos de Juján se plasma en un libro. (15 de noviembre de 2018). El Universo. Recuperado de https://www. eluniverso.com/noticias/2018/11/25/ nota/7066911/danza-mojigos-jujanse-plasma-libro

Lajo, J. (2005). Qhapaq Ñan: La ruta inka de la sabiduria. Lima: Centro de Estudios Nueva Economía y Sociedad -CENES.

Libro recoge tradicional baile de los Mojigos (26 de noviembre 2018). El Tiempo. Recuperado de https://www. eltiempo.com.ec/noticias/cultura/7/ 
baile-mojigos-cultura

Manuel, J. \& Sejas, S. (2018). La diablada boliviana y el año nuevo chino. Comparación del diseño de la indumentaria y sus significados. (Trabajo de Carrera). Argentina, Universidad de Buenos Aires.

Marquez, C. (12 de septiembre de 2018). El INPC declaró que los pases del Niño son patrimonio cultural. El Comercio Recuperado de https://www. elcomercio.com/tendencias/inpcpasedelnino-patrimonio-declaratoriariobamba.html

Merizalde, M. B. (15 de abril del 2017). Los Diablos de Alangasí irrumpieron en la iglesia. El Comercio. Recuperado de: https://www.elcomercio. com/actualidad/diablos-alangasiirrumpieron-iglesia-semanasanta.html

Ministerio de Cultura y Patrimonio (2015). El Aya Uma ayuda a mantener el orden cósmico. Recuperado de: https:// www.culturaypatrimonio.gob.ec/elaya-uma-ayuda-a-mantener-el-ordencosmico/

Monje, C. (2011). Metodología de la investigación cuantitativa y cualitativa. Guía didáctica. Universidad Surcolombiana, Colombia. Recuperado de https:// www.uv.mx/rmipe/files/2017/02/ Guia-didactica-metodologia-de-lainvestigacion.pdf

Museo Intiñan (2019). Diablo Huma. Recuperado de http:// museointinan.com.ec/wp-content/ uploads/2015/02/SV3xFPEyFNndgf1 A6lfizxzK30YEiw7E5Dzp3DJ L0msCLqJL63aYWNU81UfWR2_ uuJx2B6k2gIjbFHDqTwAU.jpg

Palao, J. (2010). La Diablada Puneña Origen y Cambios. Perú: ElectroPuno.

Pecados y Danzantes de Camuñas.(2015). Caminando por Paredes de Escalona. Recuperado de http://museointinan. com.ec/origen/diablo-huma1690x1100/

Ramírez, M. (2018). Diablos Danzantes de Corpus Christi. La eterna guerra del bien contra el mal. Venezuela. Recuperado de http://www. fundacionciev.com/diablos-danzantesde-corpus-christi-la-eterna-guerradel-bien-contra-el-mal/

Rodríguez, V., Vera, G. \& Vargas, A. (2011). Etnografía: una mirada desde corpus teórico de la investigación cualitativa. Omnia, 17(2).

UNESCO. (2011). Los Ámbitos del Inmaterial. Recuperado de: https:// i ch.unesco.org/ doc/ src/ 01857 ES.pdf? fbclid=Iw AR $1 \mathrm{fl} 15 \mathrm{f} 4 \mathrm{CJ} \mathrm{C}_{-}$ iQpgxP_AeoO1p-lpV7M5Ca46jOw_ rsyc3BHTzHbB-iVgoPc

Vallejo, G. F. (2012). Investigación de personajes míticos en celebraciones folclóricas riobambeñas y aplicación en muñecos para niños. Recuperado d efile:///C:/Use rs/wi nFoX_1/ Downloads/88T00056\%20(5).pdf 